\title{
Structure and electronic states of a graphene double vacancy with an embedded Si dopant
}

Cite as: J. Chem. Phys. 147, 194702 (2017); https://doi.org/10.1063/1.4999779

Submitted: 10 August 2017 . Accepted: 31 October 2017 . Published Online: 17 November 2017

Reed Nieman, Adélia J. A. Aquino, Trevor P. Hardcastle, Jani Kotakoski ${ }^{\circledR}$, Toma Susi ${ }^{D}$, and Hans Lischka (D)

\section{ARTICLES YOU MAY BE INTERESTED IN}

A consistent and accurate ab initio parametrization of density functional dispersion correction (DFT-D) for the 94 elements $\mathrm{H}-\mathrm{Pu}$

The Journal of Chemical Physics 132, 154104 (2010); https://doi.org/10.1063/1.3382344

Ion beam modification of two-dimensional materials: Characterization, properties, and applications

Applied Physics Reviews 4, 011103 (2017); https://doi.org/10.1063/1.4977087

Electron radiation damage mechanisms in 2D $\mathrm{MoSe}_{2}$

Applied Physics Letters 110, 033106 (2017); https://doi.org/10.1063/1.4973809 


\title{
Structure and electronic states of a graphene double vacancy with an embedded Si dopant
}

\author{
Reed Nieman, ${ }^{1}$ Adélia J. A. Aquino, ${ }^{1,2}$ Trevor P. Hardcastle, ${ }^{3,4}$ Jani Kotakoski, ${ }^{5}$ \\ Toma Susi, ${ }^{5, a)}$ and Hans Lischka ${ }^{1,2, a)}$ \\ ${ }^{1}$ Department of Chemistry and Biochemistry, Texas Tech University, Lubbock, Texas 79409-1061, USA \\ ${ }^{2}$ School of Pharmaceutical Sciences and Technology, Tianjin University, \\ Tianjin 300072, People's Republic of China \\ ${ }^{3}$ SuperSTEM Laboratory, STFC Daresbury Campus, Daresbury WA4 4AD, United Kingdom \\ ${ }^{4}$ Faculty of Engineering, School of Chemical and Process Engineering, University of Leeds, \\ 211 Clarendon Rd., Leeds LS2 9JT, United Kingdom \\ ${ }^{5}$ Faculty of Physics, University of Vienna, Boltzmanngasse 5, A-1090 Vienna, Austria
}

(Received 10 August 2017; accepted 31 October 2017; published online 17 November 2017)

\begin{abstract}
Silicon represents a common intrinsic impurity in graphene, bonding to either three or four carbon neighbors, respectively, in a single or double carbon vacancy. We investigate the effect of the latter defect $\left(\mathrm{Si}-\mathrm{C}_{4}\right)$ on the structural and electronic properties of graphene using density functional theory. Calculations based both on molecular models and with periodic boundary conditions have been performed. The two-carbon vacancy was constructed from pyrene (pyrene-2C) which was then expanded to circumpyrene-2C. The structural characterization of these cases revealed that the ground state is slightly non-planar, with the bonding carbons displaced from the plane by up to $\pm 0.2 \AA$. This nonplanar structure was confirmed by embedding the defect into a $10 \times 8$ supercell of graphene, resulting in $0.22 \mathrm{eV}$ lower energy than the previously considered planar structure. Natural bond orbital analysis showed $\mathrm{sp}^{3}$ hybridization at the silicon atom for the non-planar structure and $\mathrm{sp}^{2} \mathrm{~d}$ hybridization for the planar structure. Atomically resolved electron energy loss spectroscopy and corresponding spectrum simulations provide a mixed picture: a flat structure provides a slightly better overall spectrum match, but a small observed pre-peak is only present in the corrugated simulation. Considering the small energy barrier between the two equivalent corrugated conformations, both structures could plausibly exist as a superposition over the experimental time scale of seconds. Published by AIP Publishing. https://doi.org/10.1063/1.4999779
\end{abstract}

\section{INTRODUCTION}

The modification of the physical properties of graphene sheets and nanoribbons, in particular the introduction of a band gap via chemical adsorption, carbon vacancies, and the incorporation of dopant species has gained a great deal of attention. ${ }^{1,2}$ Silicon is an interesting element as a dopant as it is isovalent to carbon. It is also a common intrinsic impurity in graphene sheets grown by chemical vapor deposition ${ }^{3,4}$ (CVD) and in graphene epitaxially grown by the thermal decomposition of silicon carbide. ${ }^{5,6}$ Further, dopant impurities have been shown to have significant effects on the transport properties of graphene. ${ }^{7-9}$

Silicon dopants in graphene exist predominantly in two previously identified forms: a non-planar, threefoldcoordinated silicon atom in a single carbon vacancy, referred to as $\mathrm{Si}-\mathrm{C}_{3}$, and a fourfold-coordinated silicon atom in a double carbon vacancy, called $\mathrm{Si}-\mathrm{C}_{4}$, which is thought to be planar. An experimental work from 2012 by Zhou and co-authors ${ }^{10}$ showed via simultaneous scanning transmission

a)Authors to whom correspondence should be addressed: hans.lischka@ univie.ac.at and toma.susi@univie.ac.at electron microscopy (STEM) annular dark field (ADF) imaging and electron energy loss spectroscopy (EELS) and, additionally, by density functional theory (DFT) calculations that the two forms of single silicon doped graphene differ energetically, that the silicon atom in $\mathrm{Si}-\mathrm{C}_{3}$ has $\mathrm{sp}^{3}$ orbital hybridization, and that the silicon atom in $\mathrm{Si}-\mathrm{C}_{4}$ is $\mathrm{sp}^{2} \mathrm{~d}$ hybridized. Similarly, EELS experiments combined with DFT spectrum simulations confirmed the puckering of $\mathrm{Si}$ in the $\mathrm{Si}-\mathrm{C}_{3}$ structure and supported the planarity of the $\mathrm{Si}-\mathrm{C}_{4}$ structure. ${ }^{11}$ However, a less satisfactory agreement of the simulated spectrum with the experimental one was noted in the latter case. The results suggested that d-band hybridization of $\mathrm{Si}$ was responsible for electronic density disruption found at these sites. Experiments and dynamical simulations performed on these defects have demonstrated ${ }^{12}$ how the $\mathrm{Si}-\mathrm{C}_{4}$ structure is formed when an adjacent carbon atom to silicon is removed. However, it was concluded that the $\mathrm{Si}-\mathrm{C}_{3}$ structure is more stable and can be readily reconstructed by a diffusing carbon atom.

Past investigations of the electronic structure of graphene defects have shown the usefulness of the molecule pyrene as a compact representation of the essential features of single vacancy (SV) and double vacancy (DV) defects. Using this model, chemical bonding and the manifold of 


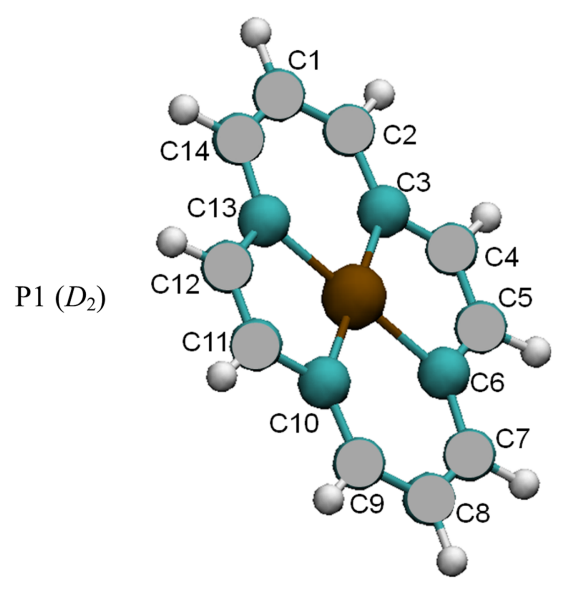

FIG. 1. Optimized pyrene-2C $+\mathrm{Si}$ structure P1 ( $D_{2}$ symmetry) using the B3LYP/6-311G(2d,1p) method with frozen carbon atoms marked by gray circles.

electronic states were investigated by multireference configuration interaction (MRCI) calculations. ${ }^{13-16}$ In the case of the SV defect, ${ }^{13}$ the calculations showed four electronic states (two singlets and two triplets) within a narrow margin of 0.1 $\mathrm{eV}$, whereas for the DV structure, ${ }^{14}$ a gap of $\sim 1 \mathrm{eV}$ to the lowest excited state was found. For the DV defect, comparison with density functional theory using the hybrid Becke threeparameter Lee, Yang, and Parr (B3-LYP) density functional ${ }^{17}$ showed good agreement with the MRCI results. Whereas for the covalently bonded $\mathrm{Si}-\mathrm{C}_{3}$ structure, no further low-lying states are to be expected, and the situation is different for the $\mathrm{Si}-\mathrm{C}_{4}$ defect since in this case an open-shell $\mathrm{Si}$ atom is inserted into a defect structure containing low-lying electronic states.

Based on these experiences, a pyrene model will also be adopted in this work to investigate the geometric arrangement and orbital hybridization around the silicon atom in a double carbon vacancy (Fig. 1). In a second step, a larger circumpyrene structure (Fig. 2) will be used to study the effect of increasing number of surrounding benzene rings. Two types of functionals will be employed, the afore-mentioned B3LYP and, for comparison, the long-range corrected Coulombattenuating B3LYP method (CAM-B3LYP) ${ }^{18}$ Special emphasis will be devoted to verifying the electronic stability of the closed-shell wavefunction with respect to triplet instability ${ }^{19,20}$ and of the optimized structures with respect to

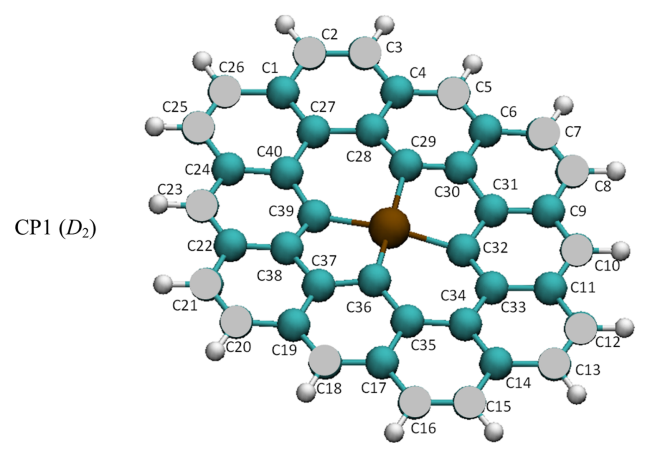

FIG. 2. Optimized circumpyrene-2C $+\mathrm{Si}$ structure $\mathrm{CP} 1\left(D_{2}\right.$ symmetry) using the B3LYP/6-311G $(2 \mathrm{~d}, 1 \mathrm{p})$ method with frozen carbon atoms marked by gray circles. structural relaxation. Finally, periodic DFT simulations of graphene with the Perdew-Burke-Ernzerhof (PBE) functional $^{21,22}$ will be compared to the molecular calculations and an EELS spectrum simulated based on the computed $\mathrm{Si}-\mathrm{C}_{4}$ structure compared to an atomic resolution STEM/EELS measurement.

\section{COMPUTATIONAL DETAILS}

Pyrene doped with a single silicon atom was investigated by first optimizing the structure of pristine pyrene using the density functional $\mathrm{B} 3 \mathrm{LYP}^{17}$ with the $6-31 \mathrm{G}^{* 23}$ basis set. The two interior carbon atoms were then removed and replaced with one silicon atom, referred to in this investigation as pyrene- $2 \mathrm{C}+\mathrm{Si}$. This unrelaxed structure was subjected to a variety of closed-shell [restricted DFT (RDFT)] and open-shell [unrestricted DFT (UDFT)] geometry optimizations using the hybrid density functionals B3LYP and CAMB3LYP in combination with the $6-311 \mathrm{G}(2 \mathrm{~d}, 1 \mathrm{p})^{24-28}$ basis set. To extend to a larger system, pyrene-2C $+\mathrm{Si}$ was surrounded by benzene rings (circumpyrene-2C) and this structure was optimized using the B3LYP/6-31G** approach. Finally, the geometry optimization of circumpyrene- $2 \mathrm{C}+\mathrm{Si}$ was performed using the B3LYP functional and the $6-311 \mathrm{G}(2 \mathrm{~d}, 1 \mathrm{~d})$ basis.

To simulate the geometric restrictions present in a large graphene sheet, the Cartesian coordinates of all carbon atoms on the outer rims of pyrene-2C $+\mathrm{Si} \mathrm{(Fig.} \mathrm{1)} \mathrm{and} \mathrm{circumpyrene-}$ $2 \mathrm{C}+\mathrm{Si}$ (Fig. 2) that are bonded to hydrogen atoms were frozen during the course of geometry optimization to their values in the pristine structures. The coordinates of both pyrene-2C $+\mathrm{Si}$ and circumpyrene-2C $+\mathrm{Si}$ were oriented along the $\mathrm{y}$-axis (the long axis) in the xy-plane.

The orbital occupations of the open-shell structures were determined using the natural orbital population analysis (NPA) ${ }^{29}$ calculated from the total density matrix, while the bonding character at the silicon for each structure was investigated using natural bond orbital analysis. ${ }^{30-34}$

The discovered corrugated ground state structure of the $\mathrm{Si}-\mathrm{C}_{4}$ defect was then introduced into a periodic $10 \times 8$ supercell of graphene, and the structure relaxed to confirm that this corrugation is reproduced at that level of theory. Finally, the Si $L_{2,3}$ EELS response of the defect was simulated ${ }^{11,35}$ by evaluating the perturbation matrix elements of transitions from the $\mathrm{Si} 2 \mathrm{p}$ core states to the unoccupied states calculated up to 3042 bands, with no explicit core hole. ${ }^{36}$ The resulting densities of state were broadened using the OptaDOS package $^{37}$ with a $0.4 \mathrm{eV}$ Gaussian instrumental broadening and semi-empirical $0.015 \mathrm{eV}$ Lorentzian lifetime broadening.

The Gaussian 09 (Ref. 38) and TURBOMOLE ${ }^{39}$ program packages were used for the molecular calculations, and the GPAW $^{40}$ and CASTEP ${ }^{41}$ packages were used for the graphene simulations.

\section{RESULTS AND DISCUSSION}

\section{A. The pyrene-2C + Si defect structure}

The geometry of the unrelaxed, planar pyrene- $2 \mathrm{C}+\mathrm{Si}$ was first optimized using the B3LYP/6-311G(2d,1p) method at the 
TABLE I. Open-shell character, structural symmetry, spin multiplicity, bond distances $(\AA)$, and relative energies $(\mathrm{eV})$ of selected optimized structures of pyrene-2C $+\mathrm{Si}$.

\begin{tabular}{|c|c|c|c|c|c|c|c|c|}
\hline Structure & $\begin{array}{l}\text { Closed/ } \\
\text { open } \\
\text { shell }\end{array}$ & $\begin{array}{c}\text { Triplet } \\
\text { instability }\end{array}$ & $\#$ \#imag $^{\mathrm{a}}$ & $\begin{array}{c}\text { High/ } \\
\text { low } \\
\text { spin }\end{array}$ & $\mathrm{Si}-\mathrm{C}^{\mathrm{b}}$ & $\mathrm{C} 3-\mathrm{C} 13$ & C3-C6 & $\Delta \mathrm{E}$ \\
\hline \multicolumn{9}{|c|}{ B3LYP/6-311G(2d,1p) } \\
\hline $\mathrm{P} 1\left(D_{2}\right)^{\mathrm{c}}$ & Closed & No & 0 & Low & 1.940 & 2.652 & 2.860 & 0.000 \\
\hline $\mathrm{P} 2\left(D_{2 h}\right)$ & Closed & No & 2 & Low & 1.932 & 2.634 & 2.826 & 0.736 \\
\hline $\mathrm{P} 3\left(D_{2 h}\right)$ & Open & $\ldots$ & 0 & High & 1.933 & 2.610 & 2.852 & 0.922 \\
\hline \multicolumn{9}{|c|}{ CAM-B3LYP/6-311G(2d,1p) } \\
\hline $\mathrm{P} 4\left(D_{2}\right)^{\mathrm{c}}$ & Open & No & 0 & Low & $1.933^{\mathrm{d}}$ & $2.645^{\mathrm{d}}$ & $2.847^{d}$ & 0.000 \\
\hline $\mathrm{P} 5\left(D_{2}\right)^{\mathrm{c}}$ & Weakly open & No & 0 & Low & 1.936 & 2.648 & 2.852 & 0.040 \\
\hline $\mathrm{P} 6\left(D_{2}\right)^{\mathrm{c}}$ & Closed & Yes & 0 & Low & 1.938 & 2.648 & 2.857 & 0.056 \\
\hline $\mathrm{P} 7\left(D_{2 h}\right)$ & Open & No & 1 & Low & 1.923 & 2.616 & 2.820 & 0.602 \\
\hline P8 $\left(D_{2 h}\right)$ & Closed & Yes & 2 & Low & 1.931 & 2.631 & 2.828 & 0.734 \\
\hline P9 $\left(D_{2}\right)^{\mathrm{e}}$ & Open & $\ldots$ & 0 & High & 1.900 & 2.599 & 2.777 & 0.770 \\
\hline $\mathrm{P} 10\left(D_{2 h}\right)$ & Open & $\ldots$ & 1 & High & 1.900 & 2.598 & 2.772 & 0.826 \\
\hline
\end{tabular}

\footnotetext{
${ }^{a}$ Number of imaginary frequencies.

${ }^{\mathrm{b}}$ Silicon always stays in-plane.

${ }^{c}$ The out-of-plane distance of carbon atoms that were not frozen in $D_{2}$ structures is $\pm 0.2 \AA$

${ }^{\mathrm{d}}$ Averaged value of the four bonding carbons.

${ }^{\mathrm{e}}$ The out-of-plane distance of carbon atoms that were not frozen is $\pm 0.07 \AA$.
}

closed-shell level resulting in the $D_{2 h}$ structure denoted as P2. There were two imaginary frequencies present in the Hessian matrix. A displacement of the geometry of structure P2 along either of these imaginary frequencies led to a distortion of the planarity, lowering the point group to $D_{2}$ and producing the minimum energy structure $\mathrm{P} 1$, which was $0.736 \mathrm{eV}$ lower in energy than structure P2 (Table I). The carbon atoms bonded to the silicon atom (atoms 3, 6, 10, and 13) were displaced from planarity by about $\pm 0.2 \AA$, while the silicon atom remained co-planar with the frozen carbon atoms on the periphery of pyrene- $2 \mathrm{C}+\mathrm{Si}$. A similar tetrahedral geometry has been found for doping $\mathrm{Al}$ and $\mathrm{Ga}$ into $\mathrm{DV}$ graphene. ${ }^{42}$ No triplet instability was found for either of the $D_{2 h}$ and $D_{2}$ structures. Optimizing the planar pyrene-2C + Si structure at the UDFT/B3LYP/6$311 \mathrm{G}(2 \mathrm{~d}, 1 \mathrm{p})$ level for the high-spin case resulted in structure $\mathrm{P} 3$, which was planar and belongs to the $D_{2 h}$ point group. No imaginary frequencies were found in the Hessian matrix of structure $\mathrm{P} 3$; it is located $0.922 \mathrm{eV}$ above the minimum energy structure P1.

Structures P1-P3 were investigated also using the CAMB3LYP/6-311G(2d,1p) method (Table I). For the planar geometry $\left(D_{2 h}\right.$ symmetry), two low-spin structures were found. The first one was the closed-shell structure P8 that, however, turned out to be triplet instable. Re-optimization at the UDFT/CAM-B3LYP level under $D_{2 h}$ restrictions led to the open-shell structure P7 that was $0.13 \mathrm{eV}$ more stable than the corresponding closed-shell structure. Structure P8 also had two imaginary vibrational frequencies, both of which were out-of-plane, leading to $D_{2}$ symmetry. Displacement along these modes and optimization at the closed-shell level gave the non-planar structure P6 of $D_{2}$ symmetry. Similarly, following the out-of-plane imaginary frequency found in the Hessian of structure $\mathrm{P} 7$ led to a new structure $\mathrm{P} 5$ ( $D_{2}$ symmetry) that was $0.016 \mathrm{eV}$ lower in energy than structure P6. It had only a small open-shell character as can be seen from a natural orbital (NO) occupation of 0.089 and $1.911 e$, respectively, for the lowest unoccupied natural orbital (LUNO) and highest occupied natural orbital (HONO). This is much smaller than the NO occupation of the open-shell low-spin structure P7 whose LUNO-HONO occupations were 0.302 and $1.698 e$. Searching along the imaginary frequencies found for the previous structures and considering the triplet instability present in the wavefunctions led to structure P4 ( $D_{2}$ symmetry), the new low-spin, open-shell minimum-energy CAM-B3LYP structure which was $0.04 \mathrm{eV}$ more stable than structure P5. The HONO-LUNO occupation for structure $\mathrm{P} 4$ was $1.899 e$ and $0.101 e$, respectively. Comparing the occupations from CAMB3LYP to B3LYP shows that the range-corrected functional CAM-B3LYP presents a more varied picture than that found with the B3LYP functional. In particular, open-shell structures with non-negligible deviations of $\mathrm{NO}$ occupations from the closed-shell reference values of two and zero, respectively, were found. However, the energy differences between these different states are quite small, only a few hundredths of an $\mathrm{eV}$.

Structure P10 of Table I was obtained using the unrestricted high-spin approach in the geometry optimization. The Hessian contained one imaginary frequency and an outof-plane bending mode leading to $D_{2}$ symmetry. Following this imaginary frequency led to structure P9 that was about $0.06 \mathrm{eV}$ more stable than structure P10. The bonding carbons of this new high-spin structure were slightly out-of-plane by $0.07 \AA$.

Selected bond distances of the silicon-2C $+\mathrm{Si}$ complex are given in Table I for the B3-LYP and CAM-B3LYP results. Looking first at the distances from the silicon atom to its bonding carbon atoms computed at the B3LYP/6-311G $(2 d, 1 p)$ level, the $\mathrm{Si}-\mathrm{C}$ bond distances are found to vary by less than 
$0.01 \AA$ in the different structures. The $D_{2}$ structure has the longest $\mathrm{Si}-\mathrm{C}$ bond distance as would be expected from moving the bonding carbon atoms out-of-plane from $D_{2 h}$ to $D_{2}$ symmetry. The internuclear distance between adjacent, non-bonded carbon atoms $\mathrm{C} 3$ and $\mathrm{C} 13$ along the direction perpendicular to the long axis is $2.65 \AA$ for structure P1, which is the largest among the three structures. This is again a result of the outof-plane character of structure P1. The distance C3-C6 along the long axis of pyrene is $\sim 0.2 \AA$ longer than the perpendicular distance, reflecting the restrictions imposed by freezing the carbon atoms which would be connected to the surrounding graphene sheet (Fig. 1).

Next, we characterize the structures computed with the CAM-B3LYP/6-311G(2d,1p) method. The largest C3-C13 bond distance (Table I) is $2.65 \AA$ for the low-spin structures P5 and P6 $\left(D_{2}\right)$, while the smallest, $2.60 \AA$, is found for the high-spin, open-shell structure P10. The dependence of the $\mathrm{Si}-\mathrm{C}$ distances on the different structures and spin states is not very pronounced though the high-spin planar structures, structures P9 and P10, were found to have the smallest $\mathrm{Si}-\mathrm{C}$ and $\mathrm{C} 3-\mathrm{C} 13$ distances; the same situation that was seen using B3LYP/6-311G(2d,1p).

Comparing the above two methods for pyrene- $2 \mathrm{C}+\mathrm{Si}$, the most noticeable effect is that there is a greater open-shell character for the low-spin structures when using the CAM-B3LYP functional. The $\mathrm{Si}-\mathrm{C}$ bond distance and $\mathrm{C} 3-\mathrm{C} 13$ intranuclear distances are also slightly smaller when using the CAMB3LYP functional. But overall, agreement between the two methods is quite good.

\section{B. The circumpyrene-2C + Si defect structure}

Pyrene-2C $+\mathrm{Si}$ was then surrounded by benzene rings to create the larger circumpyrene-2C $+\mathrm{Si}$ structure (Fig. 2) as a better model for embedding the defect into a graphene sheet. The edge carbon atoms linked to hydrogen atoms were again frozen. Description of the geometry of circumpyrene$2 \mathrm{C}+\mathrm{Si}$ is presented in Table II for the B3LYP/6-311G $(2 \mathrm{~d}, 1 \mathrm{p})$ method. The geometry of the planar, unrelaxed circumpyrene$2 \mathrm{C}+\mathrm{Si}$ was optimized using a closed-shell approach that led to the $D_{2 h}$ structure CP2, which had one out-of-plane imaginary frequency. Following this mode, the non-planar $D_{2}$ symmetric structure CP1 was obtained that contained no imaginary frequencies. Optimizing the geometry of the planar, unrelaxed circumpyrene- $2 \mathrm{C}+\mathrm{Si}$ using an open-shell, high-spin approach resulted in a geometrically stable (no imaginary frequencies) planar structure CP3 with $D_{2 h}$ symmetry. The wave functions of circumpyrene- $2 \mathrm{C}+\mathrm{Si}$ structures $\mathrm{CP} 1$ and $\mathrm{CP} 2$ were triplet stable.

The $\mathrm{Si}-\mathrm{C}$ bond distances for the three investigated structures are very similar (Table II). The C29-C39 distance is largest for the non-planar, closed-shell structure $\mathrm{P} 1$ and smallest for the planar structures $\mathrm{P} 2$ and $\mathrm{P} 3$, but the differences are less than $0.03 \AA$.

The difference in energy between the non-planar and planar low-spin structures decreases markedly when the $2 \mathrm{C}+\mathrm{Si}$ defect is embedded in a larger hexagonal sheet (compare $\Delta \mathrm{E}$ values in Tables I and II). The energy difference decreases by $\sim 0.6 \mathrm{eV}$ from $0.74 \mathrm{eV}$ in pyrene-2C $+\mathrm{Si}$ to $0.12 \mathrm{eV}$ in circumpyrene- $2 \mathrm{C}+\mathrm{Si}$. Also noteworthy, when comparing pyrene- $2 \mathrm{C}+\mathrm{Si}$ and circumpyrene- $2 \mathrm{C}+\mathrm{Si}$, is that the $\mathrm{Si}-\mathrm{C}$ bond distances in circumpyrene-2C $+\mathrm{Si}$ are shorter by about $0.03 \AA$ on average for the low-spin cases. The differences in the internuclear distance between adjacent, non-bonded carbon atoms C29 and C39 (perpendicular to the long axis) and C29-C32 (parallel) is much less pronounced than in the pyrene case, which is a consequence of the more flexible embedding into the carbon network where none of the four $\mathrm{C}$ atoms surrounding $\mathrm{Si}$ is bonded to another atom which is frozen.

\section{The graphene-2C + Si defect structure}

To confirm the obtained geometry and to validate that periodic DFT simulations reproduce the observed ground state structure, the $\mathrm{Si}-\mathrm{C}_{4}$ defect with an alternating $\pm 0.2 \AA$ corrugation of the four $\mathrm{C}$ atoms was placed in a $10 \times 8$ supercell of graphene, and the structure and cell were relaxed using the PBE functional. The structural optimization preserved the out-of-plane corrugation and resulted in a total energy $0.22 \mathrm{eV}$ lower than that of the flat structure. Thus, standard DFT is able to find the correct, non-planar structure when initializing away from the flat geometry. Further, a nudged elastic band calculation ${ }^{43}$ shows that the two equivalent conformations (alternating which $\mathrm{C}$ atoms are up and which are down) are separated only by this energy barrier, leading to a Boltzmann factor of $1.7 \times 10^{-4}$ at room temperature and thus a rapid oscillation between the two equivalent structures for any reasonable vibration frequency.

TABLE II. Structural symmetry, open-shell character, spin multiplicity, bond distances $(\AA)$, and relative energies $(\mathrm{eV})$ of selected optimized structures of circumpyrene-2C $+\mathrm{Si}$.

\begin{tabular}{|c|c|c|c|c|c|c|c|c|}
\hline Structure & $\begin{array}{c}\text { Closed/ } \\
\text { open } \\
\text { shell }\end{array}$ & $\begin{array}{c}\text { Triplet } \\
\text { instability }\end{array}$ & $\#$ imag $^{\mathrm{a}}$ & $\begin{array}{c}\text { High/ } \\
\text { low } \\
\text { spin }\end{array}$ & $\mathrm{Si}-\mathrm{C}^{\mathrm{b}}$ & C29-C39 & C29-C32 & $\Delta \mathrm{E}$ \\
\hline \multicolumn{9}{|c|}{ B3LYP/6-311G(2d,1p) } \\
\hline $\mathrm{CP} 1\left(D_{2}\right)^{\mathrm{c}}$ & Closed & No & 0 & Low & 1.909 & 2.690 & 2.741 & 0.000 \\
\hline $\mathrm{CP} 2\left(D_{2 h}\right)$ & Closed & No & 1 & Low & 1.902 & 2.668 & 2.713 & 0.118 \\
\hline $\mathrm{CP} 3\left(D_{2 h}\right)$ & Open & $\ldots$ & 0 & High & 1.912 & 2.662 & 2.745 & 1.254 \\
\hline
\end{tabular}

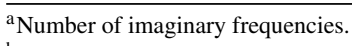

${ }^{\mathrm{b}}$ Silicon always stays in-plane.

${ }^{\mathrm{c}}$ The out-of-plane distance of non-frozen carbon atoms is $\pm 0.2 \AA$ in structures that are $D_{2}$ symmetric. 
Bader analysis ${ }^{44}$ of the charge density shows that the $\mathrm{Si}-\mathrm{C}$ bonds are rather polarized, with $\mathrm{Si}$ donating $2.61 \mathrm{elec}-$ trons shared by the four neighboring carbons. This is even larger than the bond polarization in 2D-SiC, where Si donates 1.2 electrons (although each $\mathrm{C}$ has three Si neighbors, bringing the total to 0.4 per $\mathrm{C}$ instead of 0.65 here). ${ }^{45}$

\section{Natural bond orbital analysis}

Natural bond orbital (NBO) analysis was used to further characterize the bonding of pyrene- $2 \mathrm{C}+\mathrm{Si}$ and circumpyrene$2 \mathrm{C}+\mathrm{Si}$ and to describe in detail the charge transfer from silicon to carbon in the various structures.

The NBO analysis of pyrene- $2 \mathrm{C}+\mathrm{Si}$ is presented in Table III. The linear combination factor of $\mathrm{Si}$ is less than half of that of the bonding $\mathrm{C}$, a sign of the greater electronegativity of carbon compared to silicon. The non-planar, low-spin, closed-shell structure P1 $\left(D_{2}\right.$ symmetry) shows sp ${ }^{3}$ orbital hybridization at silicon and $\mathrm{sp}^{3}$ orbital hybridization at the bonding carbon, consistent with the slight tetrahedral arrangement about the silicon center. The planar closed-shell structure $\mathrm{P} 2$ shows $\mathrm{sp}^{2} \mathrm{~d}$ hybridization at the silicon center, consistent with previous experimental and computational interpretations, ${ }^{10}$ and approximately $\mathrm{sp}^{3}$ hybridization at the bonding carbons. The high-spin structure P3, which also has $D_{2 h}$ symmetry, shows also $\mathrm{sp}^{2} \mathrm{~d}$ hybridization at silicon. Compared to B3LYP (structures P1-P3), bonding from CAM-B3LYP (Table SI of the supplementary material) is essentially the same.

The NBO analysis of circumpyrene- $2 \mathrm{C}+\mathrm{Si}$ is presented in Table IV. The bonding character of structure CP1 is essentially $\mathrm{sp}^{3}$ for both silicon and the bonding carbon as in structure $\mathrm{P} 1$ of pyrene- $2 \mathrm{C}+\mathrm{Si}$ although the $\mathrm{p}$ orbital character on the carbon atom of this structure is reduced by about $0.60 \mathrm{e}$. Structure $\mathrm{CP} 2$ presents an interesting case, as no bonding NBOs were found. Instead, a series of four unoccupied valence lone pairs $\left(\mathrm{LP}^{*}\right)$, one of s orbital character and three of $\mathrm{p}$ orbital character, were located on silicon and a corresponding set of occupied valence lone pairs (LPs), one for each bonding carbon, was found with $\mathrm{sp}^{3.58} \mathrm{~d}^{0.01}$ character. It is noted that the $\mathrm{Si}-\mathrm{C}$ bonding character of the high-spin structure $\mathrm{CP} 3$ changes markedly between the alpha shell and beta shell. In the alpha shell, both the silicon and carbon atoms exhibit $\mathrm{sp}^{1}$ orbital hybridization with no d orbital contribution on silicon, but in the beta shell, the silicon atom shows $\mathrm{sp}^{2} \mathrm{~d}$ hybridization and the carbon atoms show $\mathrm{sp}^{3}$ hybridization as in the other planar cases.

TABLE III. NBO bonding character analysis of pyrene-2C $+\mathrm{Si}$ using B3LYP/6-311G $(2 \mathrm{~d}, 1 \mathrm{p})$. The discussed carbon atom is one of the four bonded to silicon.

\begin{tabular}{lc}
\hline \hline Structure & \multicolumn{1}{c}{ Bonding character } \\
\hline & B3LYP/6-311G $(2 \mathrm{~d}, 1 \mathrm{p})$ \\
\hline P1 $\left(D_{2}\right)$ closed shell & $0.54\left(\mathrm{sp}^{2.98} \mathrm{~d}^{0.02}\right)_{\mathrm{Si}}+0.84\left(\mathrm{sp}^{3.24} \mathrm{~d}^{0.01}\right)_{\mathrm{C}}$ \\
P2 $\left(D_{2 \mathrm{~h}}\right)$ closed shell & $0.46\left(\mathrm{sp}^{2.00} \mathrm{~d}^{1.00}\right)_{\mathrm{Si}}+0.89\left(\mathrm{sp}^{2.97} \mathrm{~d}^{0.01}\right)_{\mathrm{C}}$ \\
P3 $\left(D_{2 \mathrm{~h}}\right)$ open shell, high spin & $\alpha: 0.46\left(\mathrm{sp}^{2.00} \mathrm{~d}^{1.00}\right)_{\mathrm{Si}}+0.89\left(\mathrm{sp}^{2.81} \mathrm{~d}^{0.01}\right)_{\mathrm{C}}$ \\
& $\beta: 0.55\left(\mathrm{sp}^{2.00} \mathrm{~d}^{1.00}\right)_{\mathrm{Si}}+0.83\left(\mathrm{sp}^{3.61} \mathrm{~d}^{0.01}\right)_{\mathrm{C}}$ \\
\hline
\end{tabular}

TABLE IV. NBO bonding character analysis of circumpyrene- $2 \mathrm{C}+\mathrm{Si}$. The discussed carbon atom is the one of the four bonded to silicon.

\begin{tabular}{lc}
\hline \hline Structure & \multicolumn{1}{c}{ Bonding character } \\
\hline CP1 $\left(D_{2}\right)$ closed shell & B3LYP/6-311G $(2 \mathrm{~d}, 1 \mathrm{p})$ \\
CP2 $\left(D_{2 \mathrm{~h}}\right)$ closed shell & Four LP*: one $\left[1.0(\mathrm{~s})_{\mathrm{Si}}\right]+$ three $\left[1.00(\mathrm{p})_{\mathrm{Si}}\right]^{\mathrm{a}}$ \\
& Four $\left[1.00\left(\mathrm{sp}^{3.58} \mathrm{~d}^{0.01}\right)_{\mathrm{C}}\right]^{\mathrm{b}}$ \\
CP3 $\left(D_{2 \mathrm{~h}}\right)$ open shell, & $\alpha: 0.68\left(\mathrm{sp}^{1.00} \mathrm{~d}^{0.00}\right)_{\mathrm{Si}}+0.74\left(\mathrm{sp}^{1.00} \mathrm{~d}^{0.00}\right)_{\mathrm{C}}$ \\
high spin & $\beta: 0.55\left(\mathrm{sp}^{2.00} \mathrm{~d}^{1.00}\right)_{\mathrm{Si}}+0.84\left(\mathrm{sp}^{3.41} \mathrm{~d}^{0.01}\right)_{\mathrm{C}}$ \\
\hline \hline
\end{tabular}

${ }^{\mathrm{a}}$ Four unfilled valence lone pairs (LP*) on silicon (occupation less than $1 e$ ).

${ }^{\mathrm{b}}$ One occupied valence lone pair on each bonding carbon.

The shapes of the NBOs describing the bond between $\mathrm{Si}$ and $\mathrm{C}$ are depicted in Fig. 3 for structures P1 $\left(D_{2}\right.$ symmetry) and $\mathrm{P} 2\left(D_{2 h}\right.$ symmetry) using the B3LYP/6-311G $(2 \mathrm{~d}, 1 \mathrm{p})$ method. They appear very similar in spite of the different hybridization on $\mathrm{Si}\left(\mathrm{sp}^{3}\right.$ vs. $\mathrm{sp}^{2} \mathrm{~d}$, Table III). The plots of the NBOs for all other structures, even those for circumpyrene-2C $+\mathrm{Si}$, are almost identical. The exception is structure $\mathrm{CP} 2$ of circumpyrene-2C $+\mathrm{Si}$ in which no $\mathrm{Si}-\mathrm{C}$ bonds were found during the NBO analysis. The NBOs shown in Fig. S1 of the supplementary material for this structure consist of the valence lone pairs found on each carbon atom (Table IV) possessing $\mathrm{sp}^{3.58}$ orbital hybridization with a lobe of electron density

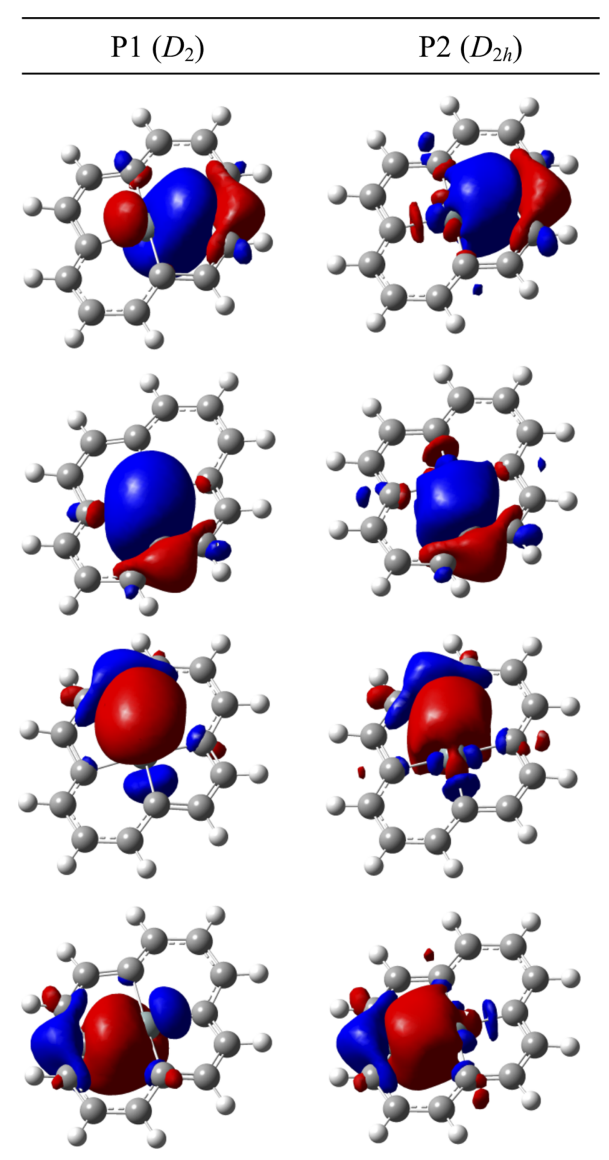

FIG. 3. NBO plots for pyrene-2C $+\mathrm{Si}$ using the B3LYP/6-311G $(2 \mathrm{~d}, 1 \mathrm{p})$ approach for structures $\mathrm{P} 1$ and $\mathrm{P} 2$. Isovalue $= \pm 0.02 \mathrm{e} / \mathrm{Bohr}^{3}$. 
found facing the silicon center. The NBOs of silicon are lowoccupancy valence lone pairs, representing one $s$ and three $p$ NBOs. Even though this classification of bonding by means of lone pairs according to the NBO analysis looks quite different, the combination of these lone pairs is not expected to look substantially different from the localized bonds shown in the other structures, especially considering the strong polarity of the silicon-carbon bonds as discussed below.

Natural charges are instructive for illustrating the polarity of the silicon-carbon bonds and the charge transfer between silicon and the surrounding graphitic lattice. These are shown in Fig. 4 for circumpyrene-2C + Si (structures CP2 and CP3) using the B3LYP/6-311G(2d,1p) method. The silicon center is strongly positive in all cases and more positive in circumpyrene-2C $+\mathrm{Si}$ for the low-spin cases (1.7-1.8 $e$, structures $\mathrm{CP} 1$ and $\mathrm{CP} 2)$ than the high-spin case (1.4 e, structure $\mathrm{CP} 3)$. It thus seems that the Bader analysis presented above overestimates the charge transfer by over $40 \%$, despite being qualitatively correct. The bonding carbon in each case shows a larger negative charge compared to the remaining carbon atoms of circumpyrene. The charge on the bonding carbon in the low-spin structures CP1 and CP2 is more negative than that of the high-spin structure CP3.

The natural charges of the remaining carbon atoms in circumpyrene-2C vary depending on whether they are bonded to other carbon atoms or to hydrogen. For the low-spin structures $\mathrm{CP} 1$ and $\mathrm{CP} 2$, the natural charges on $\mathrm{C}$ atoms on the periphery of circumpyrene bonded to other $\mathrm{C}$ atoms is about one-third as negative as the natural charges of $\mathrm{C}$ atoms bonded to $\mathrm{H}$ atoms, while the natural charges of carbon atoms in the interior of circumpyrene and not bonded to $\mathrm{Si}$ are much smaller. Taken together, the magnitude of the Si-graphene charge transfer can be seen to be largest for the planar, closedshell, low-spin structure CP2 and smallest for the planar, high-spin structure CP3.

The natural charges for pyrene-2C + Si using B3LYP/6$311 \mathrm{G}(2 \mathrm{~d}, 1 \mathrm{p})$ are plotted in Fig. S2 of the supplementary material. The low-spin structures $\mathrm{P} 1$ and $\mathrm{P} 2$ have respective positive charges on the silicon atom of 1.596 and $1.953 e$, a larger difference than in the low-spin structures CP1 and CP2 of circumpyrene-2C $+\mathrm{Si}$ where the silicon atom has positive charges of 1.726 and $1.821 \mathrm{e}$. Correspondingly, the difference in the negative charges of the bonding carbon atoms is also larger between these two structures than in circumpyrene. For the other carbons in pyrene, the magnitude of their charges varies less than in circumpyrene. For pyrene$2 \mathrm{C}+\mathrm{Si}$ using the CAM-B3LYP/6-311G(2d,1p) method, the natural charges are plotted in Fig. S3 of the supplementary material. The same trend is seen for this method as in our other cases, namely, that the closed-shell, low-spin planar structure P8 has the largest positive charge on silicon and the largest negative charge on the bonding carbon compared to the other structures.

\section{E. Molecular electrostatic potential}

The molecular electrostatic potential (MEP) was computed for each structure and is presented in Fig. 5
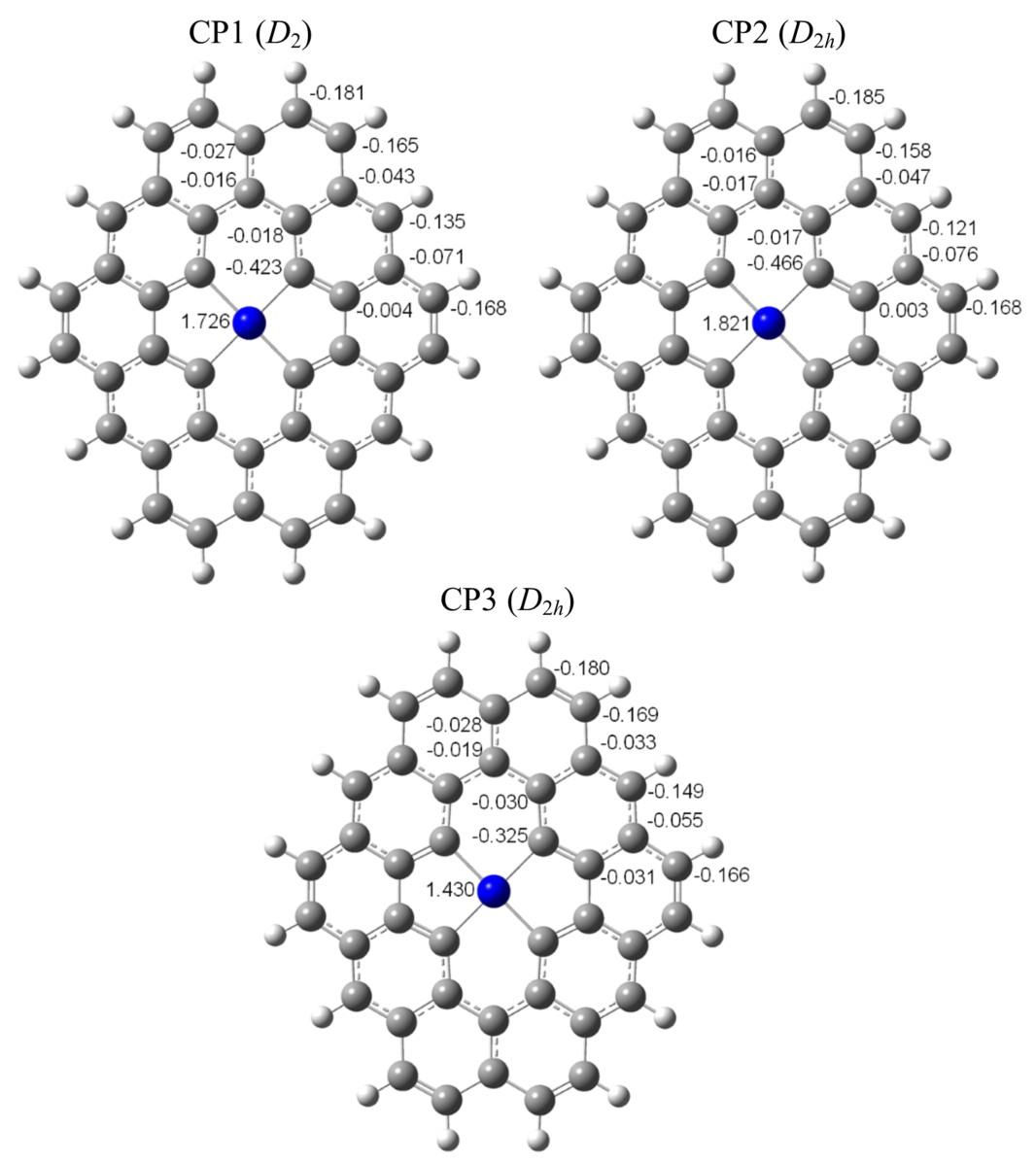

FIG. 4. Natural charges (e) of carbon and silicon in circumpyrene-2C + Si using the B3LYP/6-311G(2d,1p) method. 

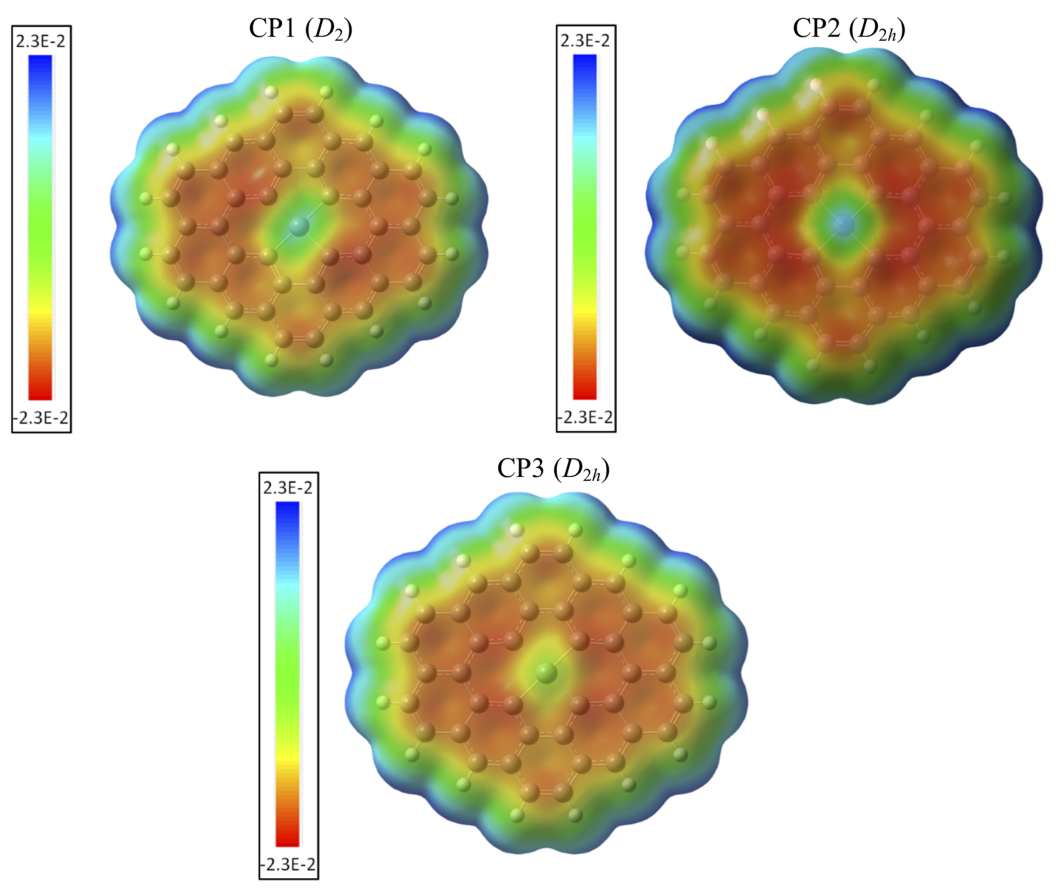

for circumpyrene-2C $+\mathrm{Si}$ using the B3LYP/6-311G(2d,1p) method. The plots illustrate the natural charge transfer discussed previously: for structures CP1-CP3, the positive potential at silicon corresponds to the positive charge buildup as discussed above. This positive potential at silicon is more positive for structures $\mathrm{CP} 1$ and $\mathrm{CP} 2$ than for CP3 following the trend of the natural charges. There is also a negative potential diffusely distributed on the carbon atoms in circumpyrene.

The MEP plots for pyrene-2C + Si using the B3LYP/6$311 \mathrm{G}(2 \mathrm{~d}, 1 \mathrm{p})$ method is shown in Fig. S4 of the supplementary material. Similar results are obtained when using the CAM-B3LYP functional (shown in Fig. S5 of the supplementary material). Finally, the low-spin planar structures $\mathrm{P} 2, \mathrm{P} 7$, and $\mathrm{P} 8$ have a much more positive potential than either the non-planar structures or the high-spin planar structures, represented by the deeper blue coloring in the figures.

\section{F. Electron energy loss spectrum}

The $\mathrm{Si}-\mathrm{C}_{4}$ defect in graphene was originally identified through a combination of atomic resolution STEM and atomically resolved EELS. In that study, ${ }^{11}$ it was concluded that a $\mathrm{Si}-\mathrm{C}_{3}$ defect is non-planar due to a significantly better match between the measured and simulated EELS spectra; however, the simulated spectrum of a flat $\mathrm{Si}_{-} \mathrm{C}_{4}$ defect matched the experiment less well. Because of this discrepancy and the findings described above, we also simulated the EELS spectrum of the corrugated $\mathrm{Si}-\mathrm{C}_{4}$ graphene defect. Figure 6 displays the simulated spectra of both the flat and the corrugated defect (normalized to the $\pi^{*}$ peak at $100 \mathrm{eV}$ ), overlaid on a new background-subtracted experimental signal that we have recorded with a higher dispersion than the original spectrum ${ }^{11}$ (average of 20 spot spectra, with the $\mathrm{Si}-\mathrm{C}_{4}$ structure verified after acquisition by imaging; see
Fig. S6 of the supplementary material for the unprocessed spectrum).

The new spectrum closely resembles the originally reported one apart from a small additional peak around $96 \mathrm{eV}$. Comparing the recorded signal to the simulated spectra, the overall relative intensities of the $\pi^{*}$ and $\sigma^{*}$ contributions match the flat structure better, but both simulations overestimate the $\pi^{*}$ intensity at $100 \mathrm{eV}$. Intriguingly, despite the better overall match with the flat structure, the small pre-peak present in the new signal is only present in the spectrum simulated for the lowest energy corrugated structure, providing

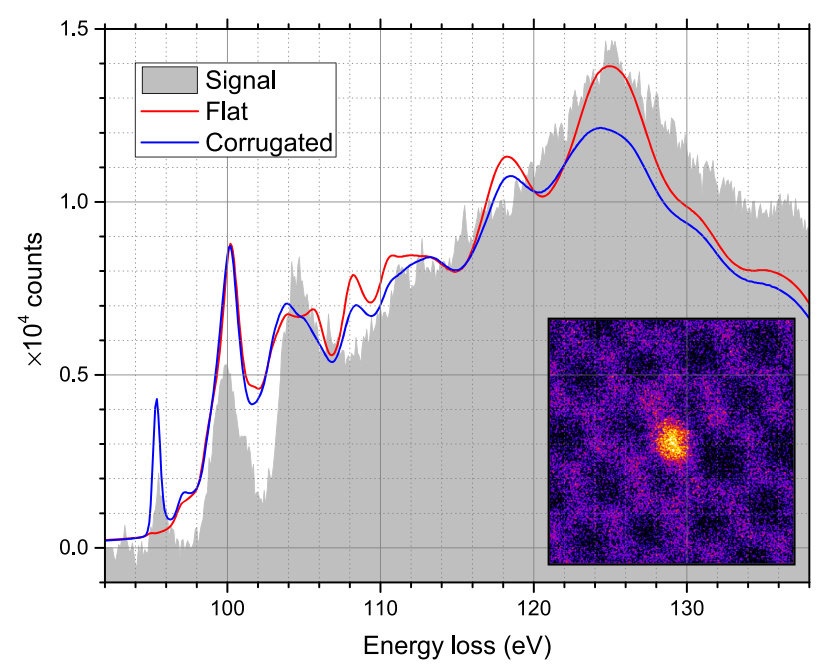

FIG. 6. Comparison of simulated and experimental EELS spectra of the Si$\mathrm{C}_{4}$ defect in graphene. Despite the out-of-plane distorted structure being the ground state, the originally proposed flat spectrum provides an overall better match to the experiment, apart from the small peak at $\sim 96 \mathrm{eV}$ that is not predicted for the flat structure. The inset shows a colored medium angle annular dark field STEM image of the $\mathrm{Si}-\mathrm{C}_{4}$ defect (the $\mathrm{Si}$ atom is brighter due to its greater ability to scatter the imaging electrons). 
spectroscopic evidence for its existence. The lowest unoccupied states responsible for this peak are $\pi^{*}$ in character and form a "cross-like" pattern across the Si site, as shown in Fig. S7 of the supplementary material, markedly different from the lowest unoccupied states of the flat structure. However, considering the small energy barrier between the equivalent corrugated conformations discussed above, even at room temperature the spectrum may be integrated over a superposition of corrugated and nearly flat morphologies.

\section{CONCLUSIONS}

Density functional theory calculations (both restricted and unrestricted) were performed on pyrene-2C $+\mathrm{Si}$ and circumpyrene-2C $+\mathrm{Si}$ producing a variety of structures that were characterized using natural orbital occupations, natural bond orbital analyses, and molecular electrostatic potential plots. A non-planar low-spin structure of $D_{2}$ symmetry was found to be the minimum for both cases, followed energetically by a low-spin planar structure, $0.6 \mathrm{eV}$ higher in energy for pyrene- $2 \mathrm{C}+\mathrm{Si}$, but with a much smaller (only $\sim 0.1 \mathrm{eV}$ ) energy difference for circumpyrene-2C $+\mathrm{Si}$. A periodic graphene model with the non-planar defect had a $0.22 \mathrm{eV}$ lower energy than a flat one. In the molecular calculations, the out-ofplane structure was shown to be a minimum by means of harmonic frequency calculations. The structures of the highspin state are much higher in energy than the minimum: about $0.85 \mathrm{eV}$ higher for pyrene-2C + Si and about $1.3 \mathrm{eV}$ higher for circumpyrene- $2 \mathrm{C}+\mathrm{Si}$.

For pyrene-2C + Si, B3LYP and CAM-B3LYP were compared showing that there was not much difference energetically either in the NBO characterization or in the MEP plots. One feature of interest is that CAM-B3LYP shows greater openshell character in the low-spin case than was seen for B3LYP. This open-shell character demonstrates the variety of electronic structures which can be found in seemingly ordinary closed-shell cases.

NBO analyses showed the expected bonding configurations for the two structural symmetries present. The bonding of the silicon atom in the planar structure was found to have $\mathrm{sp}^{2} \mathrm{~d}$ orbital character, while in the non-planar structures, the bonding orbitals are $\mathrm{sp}^{3}$ hybridized. The natural charges illustrated the charge transfer that occurs, demonstrating the buildup of positive charge at silicon and the diffusion of negative charge into the pyrene-2C/circumpyrene2C structure, which was further evident in the MEP plots.

Despite carefully establishing the non-planar ground state nature of the $\mathrm{Si}-\mathrm{C}_{4}$ defect in graphene, electron energy loss spectra still seem to match the flat structure better, but imperfectly especially concerning a small peak at $\sim 96 \mathrm{eV}$. Since the electrons used in transmission electron microscopy can impart large amounts of kinetic energy in addition to the available thermal energy, perhaps the defect is not able to stay in its ground state, but rather exists in a superposition of slightly different configurations that result in an average spectrum resembling the flat state.

\section{SUPPLEMENTARY MATERIAL}

See supplementary material for NBO analysis, NPA charges, MEP for the pyrene-2C $+\mathrm{Si}$ structures, lowest unoccupied states of the graphene $\mathrm{Si}-\mathrm{C}_{4}$ defect, and Cartesian coordinates for the different optimized structures.

\section{ACKNOWLEDGMENTS}

We are grateful to Michael Zehetbauer for continuous support and interest in this work and also thank Quentin Ramasse for many useful discussions. T.S. and J.K. acknowledge the Austrian Science Fund (FWF) for funding via Project Nos. P 28322-N36 and I 3181-N36. H.L. and T.S. are grateful to the Vienna Scientific Cluster (Austria) for computational resources (H.L.: Project No. P70376). H.L. also acknowledges computer time at the computer cluster of the School for Pharmaceutical Science and Technology of the Tianjin University, China, and T.P.H. acknowledges the Engineering and Physical Sciences Research Council (EPSRC) Doctoral Prize Fellowship that funded this research and the ARC1 and ARC2 high-performance computing facilities at the University of Leeds.

${ }^{1}$ E. Bekyarova, M. E. Itkis, P. Ramesh, C. Berger, M. Sprinkle, W. A. De Heer, and R. C. Haddon, J. Am. Chem. Soc. 131, 1336 (2009).

${ }^{2}$ E. Bekyarova, S. Sarkar, F. Wang, M. E. Itkis, I. Kalinina, X. Tian, and R. C. Haddon, Acc. Chem. Res. 46, 65 (2013).

${ }^{3}$ K. S. Kim, Y. Zhao, H. Jang, S. Y. Lee, J. M. Kim, K. S. Kim, J. H. Ahn, P. Kim, J. Y. Choi, and B. H. Hong, Nature 457, 706 (2009).

${ }^{4}$ Y. Lee, S. Bae, H. Jang, S. Jang, S. E. Zhu, S. H. Sim, Y. I. Song, B. H. Hong, and J. H. Ahn, Nano Lett. 10, 490 (2010).

${ }^{5}$ K. V. Emtsev, A. Bostwick, K. Horn, J. Jobst, G. L. Kellogg, L. Ley, J. L. McChesney, T. Ohta, S. A. Reshanov, J. Rohrl, E. Rotenberg, A. K. Schmid, D. Waldmann, H. B. Weber, and T. Seyller, Nat. Mater. 8, 203 (2009).

${ }^{6}$ C. Berger, Z. Song, T. Li, X. Li, A. Y. Ogbazghi, R. Feng, Z. Dai, A. N. Marchenkov, E. H. Conrad, P. N. First, and W. A. de Heer, J. Phys. Chem. B 108, 19912 (2004).

${ }^{7}$ A. H. Castro Neto, F. Guinea, N. M. R. Peres, K. S. Novoselov, and A. K. Geim, Rev. Mod. Phys. 81, 109 (2009).

${ }^{8}$ G. M. Rutter, J. N. Crain, N. P. Guisinger, T. Li, P. N. First, and J. A. Stroscio, Science 317, 219 (2007).

${ }^{9}$ L. Zhao, R. He, K. T. Rim, T. Schiros, K. S. Kim, H. Zhou, C. Gutiérrez, S. P. Chockalingam, C. J. Arguello, L. Pálová, D. Nordlund, M. S. Hybertsen, D. R. Reichman, T. F. Heinz, P. Kim, A. Pinczuk, G. W. Flynn, and A. N. Pasupathy, Science 333, 999 (2011).

${ }^{10}$ W. Zhou, M. D. Kapetanakis, M. P. Prange, S. T. Pantelides, S. J. Pennycook, and J. C. Idrobo, Phys. Rev. Lett. 109, 206803 (2012).

${ }^{11}$ Q. M. Ramasse, C. R. Seabourne, D. M. Kepaptsoglou, R. Zan, U. Bangert, and A. J. Scott, Nano Lett. 13, 4989 (2013).

${ }^{12}$ T. Susi, J. Kotakoski, D. Kepaptsoglou, C. Mangler, T. C. Lovejoy, O. L. Krivanek, R. Zan, U. Bangert, P. Ayala, J. C. Meyer, and Q. Ramasse, Phys. Rev. Lett. 113, 115501 (2014).

${ }^{13}$ F. B. C. Machado, J. A. J. Aquino, and H. Lischka, ChemPhysChem. 15, 3334 (2014).

${ }^{14}$ F. B. C. Machado, A. Aquino, and H. Lischka, Phys. Chem. Chem. Phys. 17, 12778 (2015).

${ }^{15}$ S. Haldar, R. G. Amorim, B. Sanyal, R. H. Scheichera, and A. R. Rocha, RSC Adv. 6, 6702 (2016).

${ }^{16}$ R. Nieman, A. Das, A. J. A. Aquino, R. G. Amorim, F. B. C. Machado, and H. Lischka, Chem. Phys. 482, 346 (2017).

${ }^{17}$ A. D. Becke, J. Chem. Phys. 98, 5648 (1993).

${ }^{18}$ T. Yanai, D. P. Tew, and N. C. Handy, Chem. Phys. Lett. 393, 51 (2004).

${ }^{19}$ R. Seeger and J. A. Pople, J. Chem. Phys. 66, 3045 (1977).

${ }^{20} \mathrm{R}$. Bauernschmitt and R. Ahlrichs, J. Chem. Phys. 104, 9047 (1996).

${ }^{21}$ J. P. Perdew, K. Burke, and M. Ernzerhof, Phys. Rev. Lett. 77, 3865 (1996).

${ }^{22}$ J. P. Perdew, K. Burke, and M. Ernzerhof, Phys. Rev. Lett. 78, 1396 (1997).

${ }^{23}$ W. J. Hehre, J. Chem. Phys. 56, 2257 (1972). 
${ }^{24}$ R. C. Binning and L. A. Curtiss, J. Comput. Chem. 11, 1206 (1990).

${ }^{25}$ L. A. Curtiss, M. P. McGrath, J. P. Blaudeau, N. E. Davis, R. C. Binning, and L. Radom, J. Chem. Phys. 103, 6104 (1995).

${ }^{26}$ R. Krishnan, J. S. Binkley, R. Seeger, and J. A. Pople, J. Chem. Phys. 72, 650 (1980).

${ }^{27}$ M. P. McGrath and L. Radom, J. Chem. Phys. 94, 511 (1991).

${ }^{28}$ A. D. McLean and G. S. Chandler, J. Chem. Phys. 72, 5639 (1980).

${ }^{29}$ A. E. Reed, R. B. Weinstock, and F. Weinhold, J. Chem. Phys. 83, 735 (1985).

${ }^{30}$ J. E. Carpenter and F. Weinhold, J. Mol. Struct.: THEOCHEM 169, 41 (1988).

31 J. P. Foster and F. Weinhold, J. Am. Chem. Soc. 102, 7211 (1980).

${ }^{32}$ A. E. Reed, L. A. Curtiss, and F. Weinhold, Chem. Rev. 88, 899 (1988).

${ }^{33}$ A. E. Reed and F. Weinhold, J. Chem. Phys. 78, 4066 (1983).

${ }^{34}$ A. E. Reed and F. Weinhold, J. Chem. Phys. 83, 1736 (1985).

${ }^{35}$ T. Susi, T. P. Hardcastle, H. Hofsäss, A. Mittelberger, T. J. Pennycook, C. Mangler, R. Drummond-Brydson, A. J. Scott, J. C. Meyer, and J. Kotakoski, 2D Materials 4, 021013 (2017).

${ }^{36}$ T. P. Hardcastle, C. R. Seabourne, D. M. Kepaptsoglou, T. Susi, R. J. Nicholls, R. M. D. Brydson, A. J. Scott, and Q. M. Ramasse, J. Phys.: Condens. Matter 29, 225303 (2017).

${ }^{37}$ R. J. Nicholls, A. J. Morris, C. J. Pickard, and J. R. Yates, J. Phys.: Conf. Ser. 371, 012062 (2012).

${ }^{38}$ M. J. Frisch, G. W. Trucks, H. B. Schlegel, G. E. Scuseria, M. A. Robb, J. R. Cheeseman, G. Scalmani, V. Barone, B. Mennucci, G. A. Petersson, H. Nakatsuji, M. Caricato, X. Li, H. P. Hratchian, A. F. Izmaylov, J. Bloino, G. Zheng, J. L. Sonnenberg, M. Hada, M. Ehara, K. Toyota, R. Fukuda, J. Hasegawa, M. Ishida, T. Nakajima, Y. Honda, O. Kitao,
H. Nakai, T. Vreven, J. A. Montgomery, Jr., J. E. Peralta, F. Ogliaro, M. J. Bearpark, J. Heyd, E. N. Brothers, K. N. Kudin, V. N. Staroverov, R. Kobayashi, J. Normand, K. Raghavachari, A. P. Rendell, J. C. Burant, S. S. Iyengar, J. Tomasi, M. Cossi, N. Rega, N. J. Millam, M. Klene, J. E. Knox, J. B. Cross, V. Bakken, C. Adamo, J. Jaramillo, R. Gomperts, R. E. Stratmann, O. Yazyev, A. J. Austin, R. Cammi, C. Pomelli, J. W. Ochterski, R. L. Martin, K. Morokuma, V. G. Zakrzewski, G. A. Voth, P. Salvador, J. J. Dannenberg, S. Dapprich, A. D. Daniels, Ö. Farkas, J. B. Foresman, J. V. Ortiz, J. Cioslowski, and D. J. Fox, Gaussian 09, Revision E.01, Gaussian, Inc., Wallingford, CT, USA, 2009.

${ }^{39}$ R. Ahlrichs, M. Bär, M. Häser, H. Horn, and C. Kölmel, Chem. Phys. Lett. 162, 165 (1989).

${ }^{40}$ J. Enkovaara, C. Rostgaard, J. J. Mortensen, J. Chen, M. Dulak, L. Ferrighi, J. Gavnholt, C. Glinsvad, V. Haikola, H. A. Hansen, H. H. Kristoffersen, M. Kuisma, A. H. Larsen, L. Lehtovaara, M. Ljungberg, O. Lopez-Acevedo, P. G. Moses, J. Ojanen, T. Olsen, V. Petzold, N. A. Romero, J. StausholmMøller, M. Strange, G. A. Tritsaris, M. Vanin, M. Walter, B. Hammer, H. Häkkinen, G. K. H. Madsen, R. M. Nieminen, J. K. Nørskov, M. Puska, T. T. Rantala, J. Schiøtz, K. S. Thygesen, and K. W. Jacobsen, J. Phys.: Condens. Matter 22, 253202 (2010).

${ }^{41}$ S. J. Clark, M. D. Segall, C. J. Pickard, P. J. Hasnip, M. J. Probert, K. Refson, and M. C. Payne, Z. Kristallogr. - Cryst. Mater. 220, 567 (2005).

${ }^{42}$ L. Tsetseris, B. Wang, and S. T. Pantelides, Phys. Rev. B 89, 035411 (2014).

${ }^{43}$ G. Henkelman and H. Jónsson, J. Chem. Phys. 113, 9978 (2000).

${ }^{44}$ W. Tang, E. Sanville, and G. Henkelman, J. Phys.: Condens. Matter 21, 084204 (2009).

${ }^{45}$ T. Susi, V. Skákalová, A. Mittelberger, P. Kotrusz, M. Hulman, T. J. Pennycook, C. Mangler, J. Kotakoski, and J. C. Meyer, Sci. Rep. 7, 4399 (2017). 